\title{
A general design method for tunable microstrip devices at microwave frequency based on liquid crystal technology
}

\author{
Ming Lei
}

Department of Electronic and Electrical Engineering, University College London, London, United Kingdom

\section{Zijun Nie}

Department of Electronic and Electrical Engineering, University College London, London, United Kingdom

Mengyang Yang

Department of Electronic and Electrical Engineering, University College London, London, United Kingdom

F. Aníbal Fernández

Department of Electronic and Electrical Engineering, University College London, London, United Kingdom

Clive Poole

Department of Electronic and Electrical Engineering, University College London, London, United Kingdom

Sally E. Day

Department of Electronic and Electrical Engineering, University College London, London, United Kingdom (email: sally.day@ucl.ac.uk)

This work was supported in part by the EPSRC under grant [EP/I003894/1]. 


\section{A general design method for tunable microstrip devices at microwave frequency based on liquid crystal technology}

This paper proposes a general design method for microstrip devices using liquid crystals (LCs) at microwave frequency. The method employs lumped element modelling to first optimise the filter at the working frequency. Then EM simulation is used for the second optimisation and the full-wave simulation. LC is used as the tunable material since the anisotropy can be changed with low voltages. The approach of modelling of LC as a homogeneous anisotropy demonstrated with Computer Simulation Technology (CST) Microwave Studio is an acceptable approximation, which accurately predicts the tuning range. A new tunable inverted microstrip filter using nematic LCs at millimetre-wave frequencies is designed to verify the method. The proposed design utilises interdigital capacitors in parallel with two inductive loops to form a bandpass filter. It is fabricated and measured, and shows that the centre frequency varies from 5.01 to $5.51 \mathrm{GHz}(10 \%$ tunability) and achieves a 3-dB bandwidth of $450 \mathrm{MHz}$, which is in good agreement with simulation results. A finite-element simulation is used to investigate the detailed behaviour of LC directors in the fully switched state; the effective permittivity is extracted from the finite-element simulation and used in CST to achieve an even more accurate agreement with experimental results.

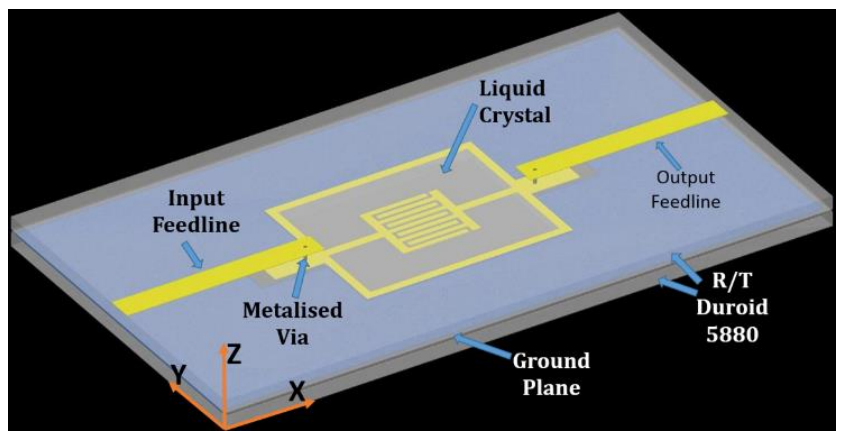

Keywords: Liquid crystals; frequency-agile; finite element modelling; inverted microstrip structure; tunable bandpass filter.

\section{Introduction}

Liquid crystals (LCs) are a promising microwave material due to their tunability and low losses at microwave frequencies [1]. LCs can be tuned by an external electric field and have the advantages of low cost, low bias voltage and very low power consumption [1]. They have been 
shown to have good capability with different types of tunable devices. However, there are some considerations and restrictions on designing LC devices, such as LC containment, alignment layer requirements and modelling the inhomogeneous anisotropy of LCs. Therefore, a proper design strategy should be used to design tunable devices using LCs at the desired frequency range.

Tunable devices, that can realise multiple allocated frequencies in a single device and achieve a tunability $\left(\frac{\Delta f_{c}}{f_{c}}\right)$ of $10 \%$ or more for multi-band, have become popular in recent years in the field of Radio Frequency Identification (RFID) [2], WiFi [3] and other frequency ranges $[4,5]$. Tunable filters can be inserted in multifunctional, multiband systems, and offer reduced size, low power consumption and a convenient tuning mechanism when compared with fixed filter solutions over the same multiple bands [6-8] while maintaining low loss operation.

Various tuning techniques have been developed for the fabrication of tunable filters [1]. MicroElectroMechanical Systems (MEMS) is the common and practical technique for mechanical tuning, and it offers fast tuning speed and high linearity [9-11]. But the reliability issues and increase in insertion loss at millimetre-wave bands are the major challenges $[9,11]$. Integrated electronic tunable devices, such as PIN diodes and varactor diodes are also used to achieve tuning and provide a wide tuning range [12, 13]; however the high loss in the microwave band is not acceptable [13]. Tunable materials, such as ferrites, ferroelectrics and semiconductors, are used to attain tuning through changing the permittivity and permeability of the materials by an external field, such as electric or magnetic fields $[14,15]$. However, material losses and the high voltage requirements are particular problems for these tunable materials used at microwave frequencies [16].

An emerging interest in the use of LC as a tunable material has been realised with the development of microwave and Terahertz LC applications in resonators [17, 18], filters [1922], phase shifters [23], tunable antennas [24], delay lines [25] and frequency selective surfaces 
[26]. In [24], the series-fed patch array is used to design antennas; it can change the operating frequency from 14.5 to $16.4 \mathrm{GHz}$. In [27] and [28], two types of tunable filters based on a dual mode structure and DBR topology are reported, and they achieve a tunability of $6 \%$ at $5 \mathrm{GHz}$ and $6 \%$ at $4 \mathrm{GHz}$, respectively. However, in simulating these tunable devices, such as in [24, $27,28]$, the LC is treated as an isotropic material, ignoring the LC anisotropy. It can be seen from much of the previous work that there is a lack of a general design method for microstrip tunable devices based on LC technology.

In this work, a design method for tunable devices using LC in a microstrip configuration is presented. The design method consists of two stages using lumped element modelling followed by full-wave simulation. In the first stage, the elements of the basic microstrip line circuit are converted to lumped element equivalents using theoretical and empirical equations. Then the lumped element equivalent circuit is modelled using Advanced Design System (ADS) [29] to optimise the element values and adjust the working frequency and the tuning range. In the second stage, the optimised elements are converted back to microstrip segments and CST Microwave Studio [30] is used for full-wave simulation of the structure. The results are compared with the lumped element modelling to make sure the initial lumped element model provides the correct estimation. The LC used in CST is approximated and defined as a homogeneous anisotropic material. If necessary, the LC region can be divided into several homogeneous parts. This approximation can be verified by comparing the electric field distribution in CST and the expected LC director distribution using LC modeling. CST is then used to optimise the dimensions of the device for the second time to get the final dimensions for the required frequency range.

To validate the design method, a new tunable microstrip bandpass filter using a voltagetunable LC layer is designed and fabricated. Measured results are found to be in good agreement with the predicted simulation results. 


\section{Design of tunable bandpass filter}

\subsection{Overview of the tunable bandpass filter}

The basic construction of the tunable device using LC is shown in Figure 1(a). The microstrip electrodes and ground plane are patterned on dielectric substrates, and the surface of the microstrip electrodes and ground plane are coated with a thin alignment layer to orient the LC. The designed tunable device is based on an inverted microstrip structure shown in Figure 1(b), and it has the feed line placed on the upper side of the top substrate, and connected to the inverted microstrip structure on the inside using metalised vias to achieve impedance matching. The LC cavity is formed by using a spacer gasket that separates the inverted microstrip structure on the inside and ground plane and also contains the LC material. With this configuration, a low frequency voltage can be applied between the microstrip lines and the ground plane to control the LC orientation. The advantage of this form of applying the biasing field is that for the maximum voltage, the director orientation and consequently, the value of epsilon parallel will occur in the direction of the RF field throughout the LC region producing the largest effect. SMA connectors are used to connect the microstrip input to the filter, and make it easier to integrate into other microwave systems.

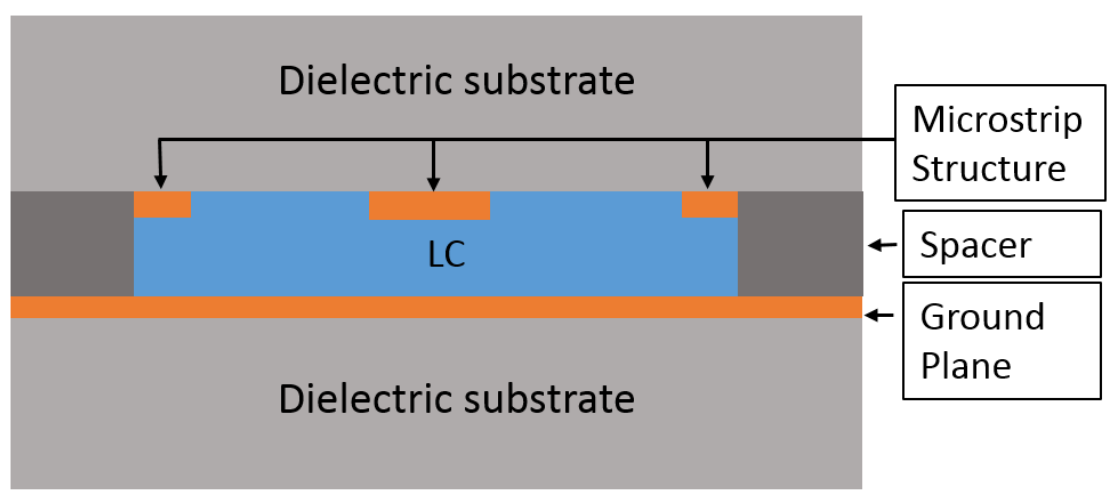




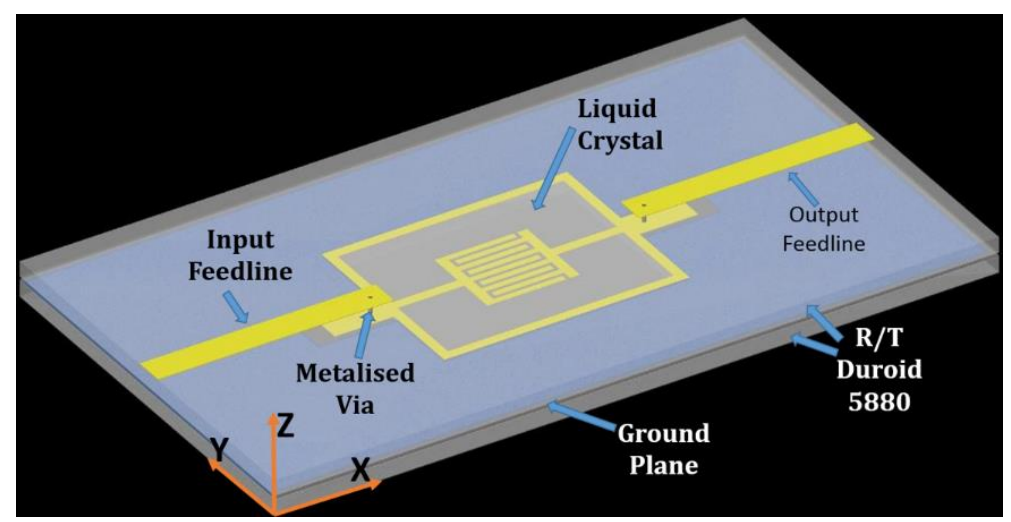

Figure 1. (a) Cross section view of the tunable filter (not drawn to scale); (b) 3D view of the inverted bandpass tunable filter
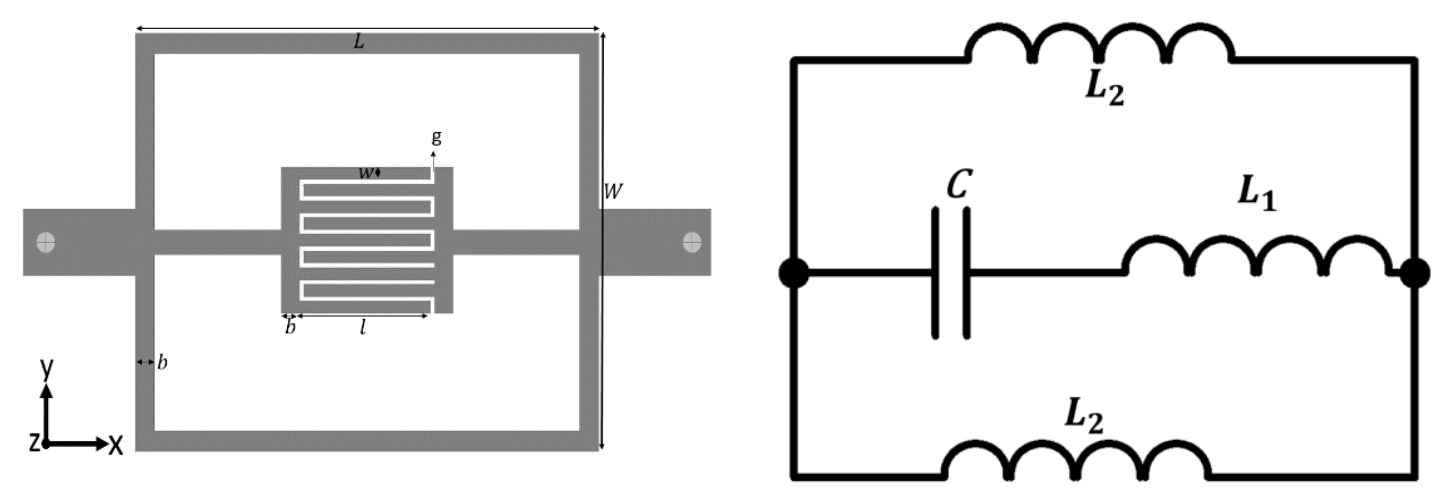

Figure 2. (a) Schematic view of the filter; (b) equivalent circuit

\subsection{Theoretical equations and lumped element modelling}

The inverted microstrip bandpass filter designed to illustrate the design approach is based on an inductive-capacitive resonant structure. The detailed structure designed is shown in Figure 2(a), and follows ideas from [31]. As long as the overall size of the filter is a small fraction of the operating wavelength, this filter can be approximated by the inductors and capacitor in the form of a resonant circuit $[32,33]$. As shown in Figure 2(a), the interdigital capacitors (IDCs) provide the capacitance and the two outer branches connecting in parallel provide the inductance to the circuit. The IDC is used to achieve an increased effective length in a small area, which results in a higher capacitance in a small area and also to decrease the amount of LC material needed, while maintaining the value of inductance from the outer loops. The 
simplified equivalent circuit for the filter is shown in Figure 2(b), where $C$ is the total equivalent capacitance of the IDC, $L_{1}$ is the inductance from the IDC and $L_{2}$ is the inductance of the loops. Compared with the total equivalent capacitance, the capacitance between electrodes and ground plane is more than 10 times smaller; therefore, it is not included in the theoretical analysis.

For the resonant circuit, $C$ and $L$ are needed to determine the resonant frequency. The partial capacitance method [34] is used to find the capacitance and inductance of the filter, and the capacitance $C$ of the IDC can be analytically calculated following [35]:

$$
C=\frac{\varepsilon_{0} \varepsilon_{e f f} 10^{-3}}{18 \pi} \frac{K(k)}{K^{\prime}(k)}(N-1) l
$$

where $K(k)$ is the elliptic integral of first kind, $K^{\prime}(k)$ is its complement, and the ratio between them is given by

$$
\frac{K(k)}{K^{\prime}(k)}= \begin{cases}\frac{1}{\pi} \ln \left\{2 \frac{1+\sqrt{k}}{1-\sqrt{k}}\right\} & \text { for } 0.707 \leq k \leq 1 \\ \frac{\pi}{\ln \left[\frac{1+\sqrt{k^{\prime}}}{1-\sqrt{k^{\prime}}}\right]} & \text { for } 0 \leq k \leq 0.707\end{cases}
$$

and $k=\tan ^{2}\left(\frac{w \pi}{4(w+g)}\right), k^{\prime}=\sqrt{1-k^{2}}$. Thus, the capacitance of the IDC can be seen as a function of finger length $l$, the total number of fingers $N$, the line width $w$, the gap $g$ and effective dielectric constant $\varepsilon_{e f f}$.

The inductance of the IDC $L_{1}$ is approximated as [35]

$$
L_{1}=\frac{Z_{o \sqrt{\varepsilon_{e f f}}}}{c}
$$

where $\varepsilon_{e f f}=\frac{\varepsilon_{r}+1}{2}+\frac{\varepsilon_{r}-1}{\sqrt{1+12\left(\frac{h}{b}\right)}}, Z_{o}$ and $\varepsilon_{e f f}$ are calculated with the loop width $b$, thickness of

LC layer $h, c$ is the velocity of light in free space.

The loop inductance $L_{2}$ is approximated as

$$
L_{2}=\mu_{0} \mu_{r} h\left(2 \frac{b}{W}+2 \frac{L}{b}+\frac{L-2 b-g-l}{b}\right)
$$

The permeability $\mu_{r}$ of the LC can be approximated by $\mu_{r}=1$. 
The resonant frequency for this filter can be calculated as

$$
f=\frac{1}{2 \pi \sqrt{\left(L_{2}+L_{1}\right) C}}
$$

This filter is designed to work at $5 \mathrm{GHz}$. Based on theoretical calculations and on fabrication considerations, such as the constraints of total area available for the filter, the minimum gap $g$ between electrodes and keeping low inductance, the largest tuning range is achieved when the number of fingers is 9. The LC used is E7, made by Merck, and its permittivity values are $\varepsilon_{\mathrm{r} \perp}=2.72$ and $\varepsilon_{r \|}=3.17$ at room temperature [1]. The lumped element simulation with ADS shows that approximating the LC as an isotropic material, the resonant frequency shifts from 5.5 to $5.03 \mathrm{GHz}$ when the (scalar) permittivity changes from 2.72 to 3.13 , and the tuning range is $480 \mathrm{MHz}$. Then the first optimisation using ADS suggests that changing the capacitance $C_{I D C}$ contributes the most to obtain the maximum tunability, and changing $L_{1}$ has the second largest effect. Therefore, in the second optimisation with CST, the length $l$, the width $w$ and the gap $g$ of the IDC are the most important dimensions that need to be optimised. Table 1 shows the dimensions of the filter after the first optimisation.

Table 1. Dimensions of the filter after ADS optimisation (in mm).

\begin{tabular}{|c|c|c|c|c|c|}
\hline$L$ & $W$ & $l$ & $w$ & $b$ & $g$ \\
\hline 12 & 12 & 3.5 & 0.25 & 0.5 & 0.2 \\
\hline
\end{tabular}

\subsection{E-field observation using CST and finite element modelling of director switching}

CST is used as the electromagnetic wave simulator to verify the resonant frequency obtained from the equivalent circuit analysis using ADS and guide the second optimisation process. For the structure shown in Figure 1(b), the material used for the dielectric substrate is the Rogers Duroid $5880\left(\varepsilon_{r}=2.20\right)$ with a thickness of $0.79 \mathrm{~mm}$. The spacer used is another piece of Rogers Duroid $5880\left(\varepsilon_{r}=2.20\right)$ with a thickness of $0.13 \mathrm{~mm}$. This material has been chosen because its low permittivity allows higher field confinement in the LC region, increasing the effect of the LC switching. CST can only deal with homogeneous blocks of isotropic or 
anisotropic material; therefore, the LC is assumed in the simulations to be a single homogeneous anisotropic layer. However, the LC switches into a non-uniform structure. In order to examine the validity of this approximation, the electric field distribution calculated with CST and the LC director distribution are compared for the case of saturation voltage (maximum switching). With no voltage applied, the LC orientation is uniform and in the direction given by the surface alignment, so the simulations are expected to be accurate in the unswitched case. The director distribution is calculated using an in-house finite element LC modelling program [36].

Figure 3 (a) and (b) shows the electric field below and between the IDC electrodes and at the edge of the IDC for the case when the maximum voltage is applied in the CST. The arrows in (a) and (b) indicate the direction of the field and their colours represent its intensity, where those in red show the highest field intensity. It can be seen that the field is largely confined between the IDC electrodes and the ground plane, and in the $z$-direction as defined in Figure 1(b). Figure 3 (c,d) shows the corresponding director distributions in the same regions as (a) and (b).

We can observe that although the LC in Figure 3(c) is almost fully switched, it is not fully vertical across the whole of the cross section, which would represent a homogeneous permittivity. Compared with Figure 3(a), the LC directors largely follow the direction of the electric field, which in fact would maximise the tuning effect by increasing the actual effective permittivity and in turn shows a larger tuning range in the measurement. However, there is a narrow layer next to the alignment layers that will remain unswitched and this will contribute to a small decrease in the effective permittivity, the assumption of uniform, vertically aligned permittivity ellipsoid here will overestimate the effective permittivity. Beyond the edge of the electrodes (Figure 3(b) and (d)), the LC orientation is not vertical and the assumption of vertically oriented permittivity will underestimate the effective permittivity. However, the field 
values in this region decrease sharply away from the electrodes so the approximation has a lower effect. Since the effect of assuming a vertically oriented permittivity in the different regions tends to compensate each other, we have considered it reasonable to use this approximation in the CST simulations.

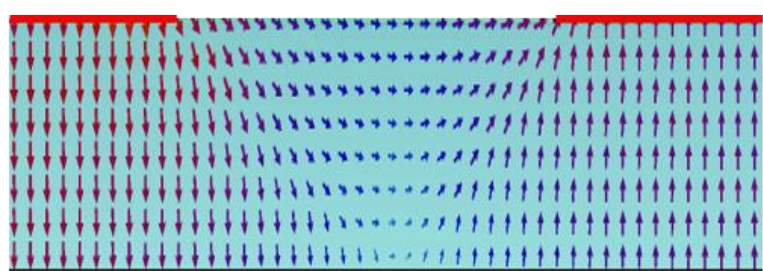

(a)

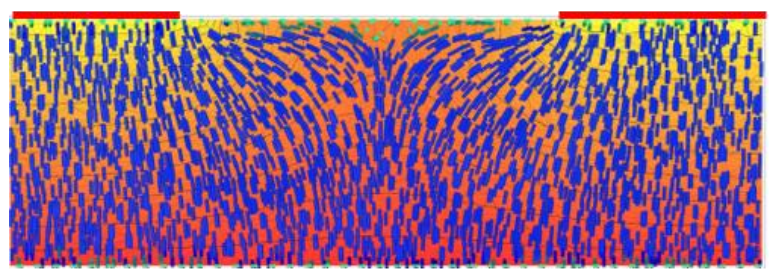

(c)

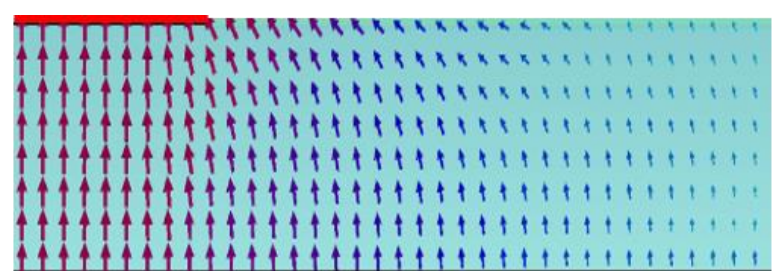

(b)

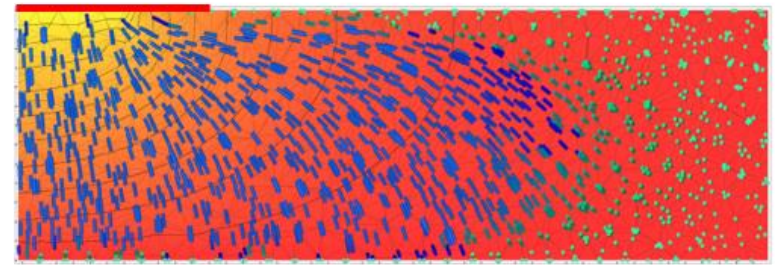

(d)

Figure 3. Electric field distribution for the saturation voltage $(16 \mathrm{~V})$ in the region between the IDC electrodes (a) and at the edge of the electrodes (b). The corresponding director distributions are shown in (c) and (d), respectively.

The direction of alignment layer, or the direction of the unswitched state, can also have an effect on the performance and needs to be chosen carefully. The unswitched orientation can be chosen as $x$ or $y$ (see Figure 1(b)) and CST modelling shows that selecting $x$ gives the maximum tuning range, as shown in Figure (5) later. Also, since the direction of the unswitched state is imposed by the rubbing of the alignment layer, from the fabrication point of view it is clear that rubbing along $x$, which is the direction along the fingers of the IDC, is more likely to produce an effective anchoring. 


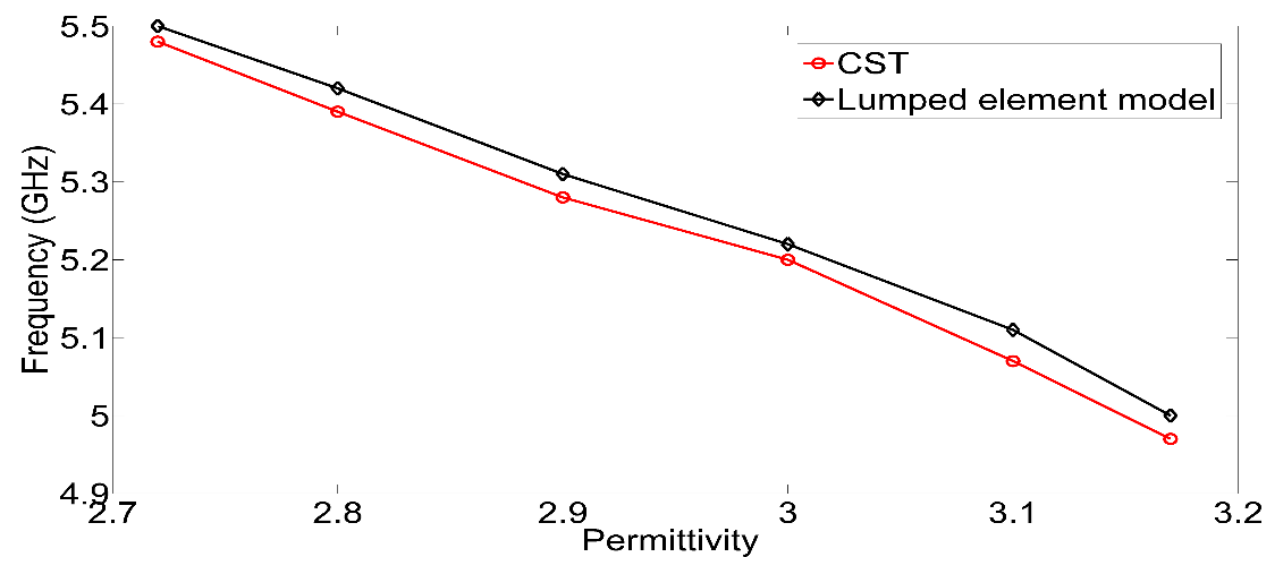

Figure 4. Simulated and theoretical resonant frequency versus $\varepsilon_{r}$.

\subsection{Second optimisation and full wave simulation using CST}

In order to make sure the lumped element model is satisfactory, a comparison between the resonant frequencies from (5) and from CST simulations is plotted versus $\varepsilon_{r}$ in Figure 4, which uses the values for the device dimensions given in Table 1. The results from CST and ADS show a good agreement and the differences are within $1 \%$. The small discrepancies between the theoretical and simulated resonant frequencies are due to the parasitic elements that are ignored in the analytical calculations with ADS.

The next optimisation stage of the design is using CST to maximise the tuning range as the permittivity ellipsoid is changed from along $x$ to along $z$ assuming the LC is homogeneous, as discussed above. The optimisation involves keeping the inductive loop fixed and varying three of the dimensions of the IDC, the length $l$, the inter-electrode gap $g$ and the width $w$. The optimisation starts by fixing the length $l$ from the initial calculation shown in Table 1 , and changing $g$ and $w$. With fixed $l$ and considering the resolution limits of fabrication, the gap is changed from 0.05 to $0.25 \mathrm{~mm}$ with steps of $0.05 \mathrm{~mm}$, and width is changed from 0.1 to 0.5 $\mathrm{mm}$ with steps of $0.05 \mathrm{~mm}$. The optimization shows that $g=0.2 \mathrm{~mm}$ and $w=0.3 \mathrm{~mm}$ give the largest tunability. Then, $g$ and $w$ are kept fixed and the length $l$ is changed with steps of 0.2 
$\mathrm{mm}$. With a length of $4 \mathrm{~mm}$, the largest tuning range is achieved, with a tunability of $11 \%$. The final optimised dimensions of the proposed filter are shown in Table 2.

Table 2. Dimensions of the filter after CST optimisation (in $\mathrm{mm}$ ).

\begin{tabular}{|c|c|c|c|c|c|}
\hline$L$ & $W$ & $l$ & $w$ & $b$ & $g$ \\
\hline 12 & 12 & 4 & 0.3 & 0.5 & 0.2 \\
\hline
\end{tabular}

The full-wave simulation results of the final optimised structures are shown in Figure 5. The resonant frequencies for alignments along $x$ and $y$ are similar, 5.52 and $5.49 \mathrm{GHz}$ respectively, with the alignment along $x$ giving the slightly larger tuning range. With alignment along $x$, the resonant frequency shifts from 5.52 to $4.97 \mathrm{GHz}$ on reorientation to the $z$-direction, as occurs with an applied voltage. The frequency shift corresponds to a tuning range of $550 \mathrm{MHz}$, and the tunability is $11 \%$.

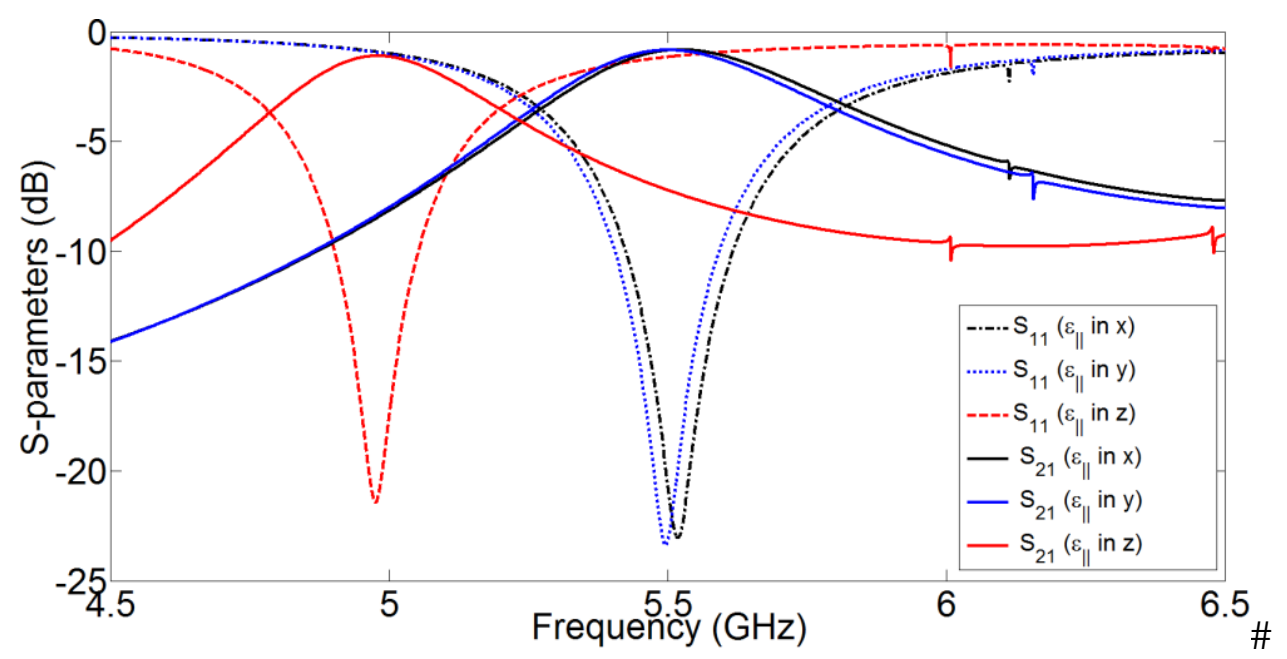

Figure 5. Simulation results of insertion loss $\left(S_{21}\right)$ and return loss $\left(S_{11}\right)$

\section{Experimental results and discussion}

\subsection{Fabrication procedure}

Standard photolithography was used to fabricate the test filter. The feedlines and the microstrip 
filter structure on the dielectric layers were masked and etched, as shown in Figure 1. In order to form the cavity to contain the LC, the surfaces must be initially prepared. The inner top and bottom surfaces of the filter were coated with a thin layer of polyvinyl alcohol (PVA) made by Sigma-Aldrich and then, mechanically rubbed longitudinally (along $x$ in Figure 1(b)) to control the alignment of the surface anchoring. The device was filled with LC using a fine syringe through a small fill hole on the bottom layer after assembly.

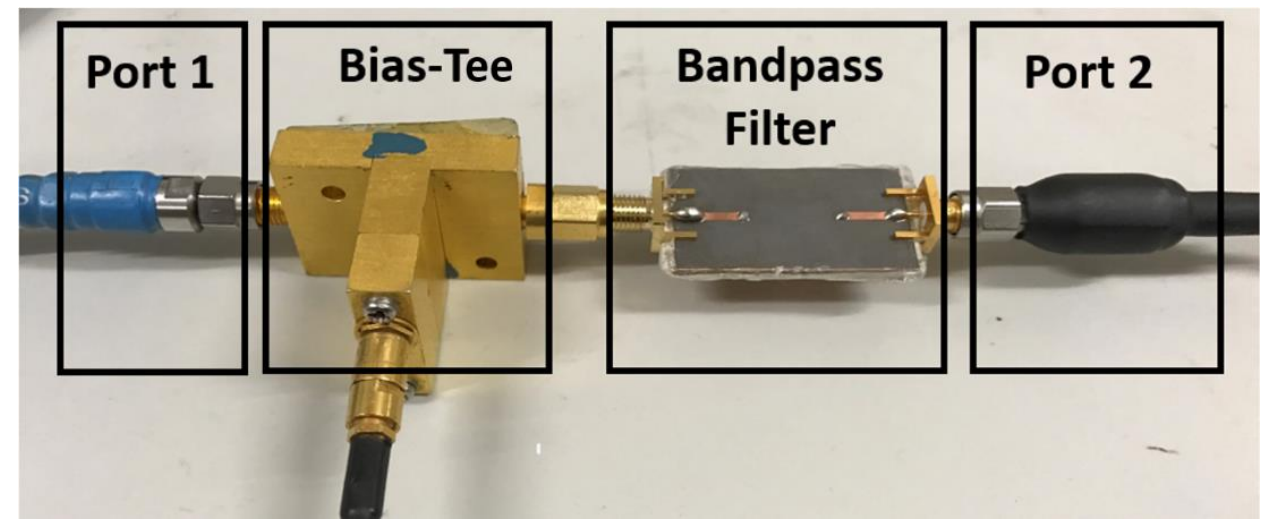

Figure 6. Measurement setup for the experiment (from left: port1, bias-tee, bandpass filter and port2).

\subsection{Measurement setup}

The measurement setup is shown in Figure 6. The frequency response was measured using a Keysight E5071C network analyser. The input port of the network analyser was connected to a bias-tee, which is used to decouple the $1 \mathrm{kHz}$ bias voltage source and the microwave signal. Measurements were taken with the biasing voltage amplitude varying from 0 to $16 \mathrm{~V}$ in $0.5 \mathrm{~V}$ steps. 

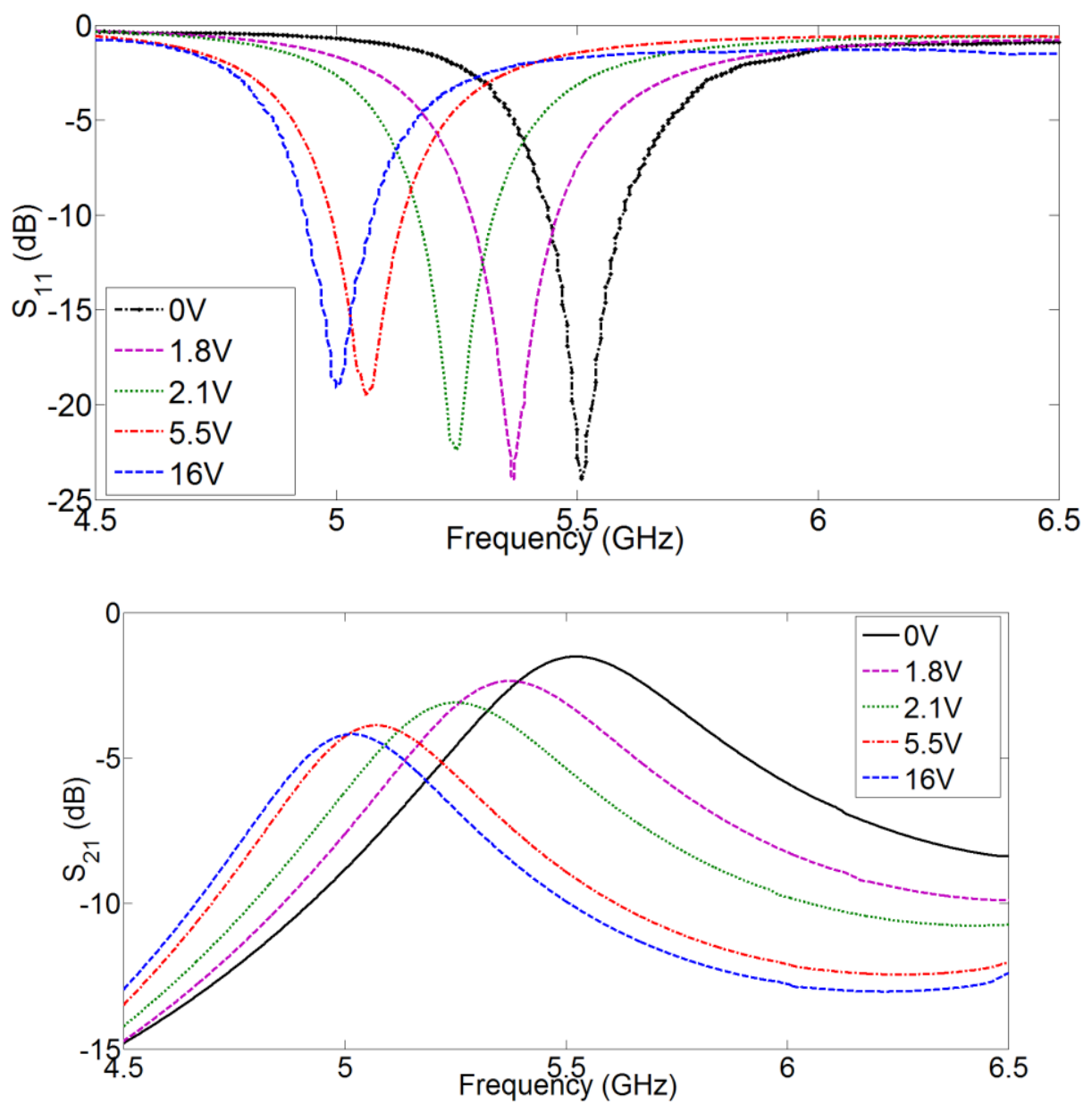

Figure 7. Measured S-parameters with changing bias voltage: (a) measured $S_{11}$ with different bias voltages; (b) measured $S_{21}$ with different bias voltage

\subsection{Measurement of the tunable filter}

The S-parameters of the filter measured at different bias voltages are shown in Figure 7. A clear shift in the resonant frequency is observed in the measured response, which indicates that increasing the bias voltage gradually switches the LC and reorients it from the unswitched state. As shown in Figure 7(a), with no bias voltage the resonant frequency is $5.51 \mathrm{GHz}$ and the FBW is $8.3 \%$. When the bias voltage reaches the saturation value of around $16 \mathrm{~V}$, the LC is largely aligned perpendicular to the electrodes (along $z$ in Fig. 1(b)). The resonant frequency reaches a minimum value of $5.01 \mathrm{GHz}$ with a FBW of $9.5 \%$. Therefore, the tuning range is $500 \mathrm{MHz}$, which corresponds to a tunability of $10 \%$. Figure $7(\mathrm{~b})$ indicates that when the bias voltage 
increases from 0 to $16 \mathrm{~V}$, the return loss is larger than $18 \mathrm{~dB}$ and the insertion loss changes from -1.5 to $-4.2 \mathrm{~dB}$. The increases in the insertion loss can be attributed to the anisotropy of the loss tangent, which is higher along the director [37] and to variation in the matching conditions at the two ports. No effort has been made to compensate the insertion loss or optimise the matching sections, as this is not the major concern in this work, but clearly, this is a route to improve the characteristics of the filter as a practical device.

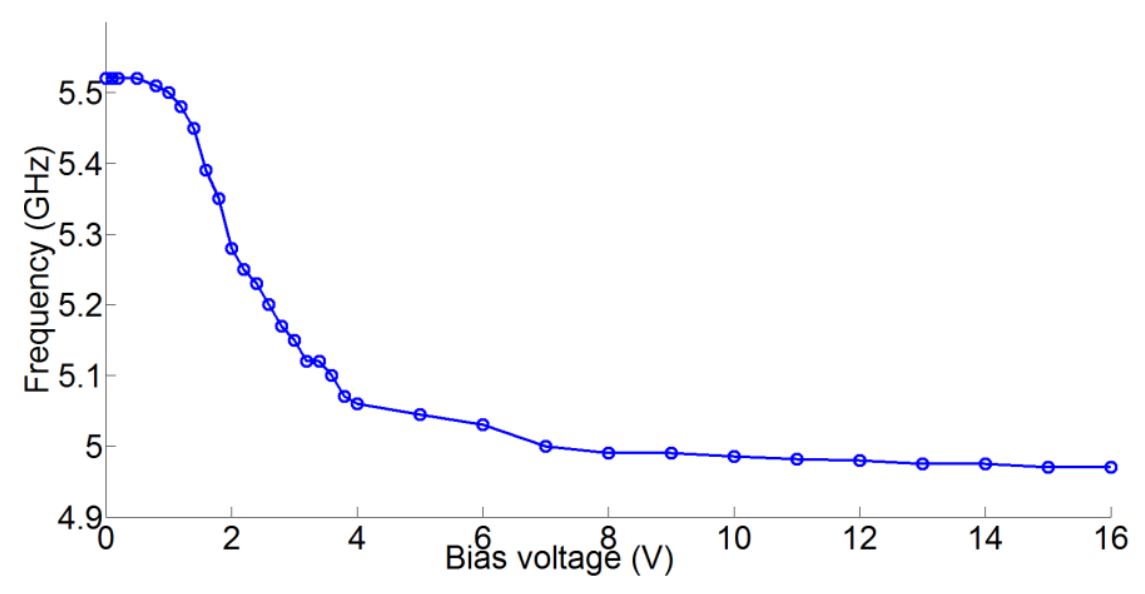

Figure 8. Centre frequency as a function of LC bias voltage

Figure 8 shows the centre frequency as a function of the bias voltage. When the voltage is applied across the LC cell the LC starts gradually to re-orient itself after the Fréedericksz transition, which for E7 is about $0.5 \mathrm{~V}$. As the voltage increases, the effective permittivity increases and the resonant frequency decreases slowly and continuously for intermediate values between 0.5 to about $7 \mathrm{~V}$ and there is little change after that. Beyond $7 \mathrm{~V}$, the LC reaches a saturation state and most of the LC is oriented perpendicular to the electrodes.

\subsection{Finite element modelling of the detailed LC switching}

Figure 9 shows the comparison of CST simulation and measurement results for the unswitched and switched states. As can be seen, the simulated and measured $\mathrm{S}_{11}$ show very good agreement in the unswitched state where the LC is uniformly aligned along $x$. However, in the switched state a small difference is observed, from $4.97 \mathrm{GHz}$ in the simulation to $5.01 \mathrm{GHz}$ for the 
measured $\mathrm{S}_{11}$. This is due to the approximation of the permittivity as a homogeneous tensor in the simulated results as discussed earlier.

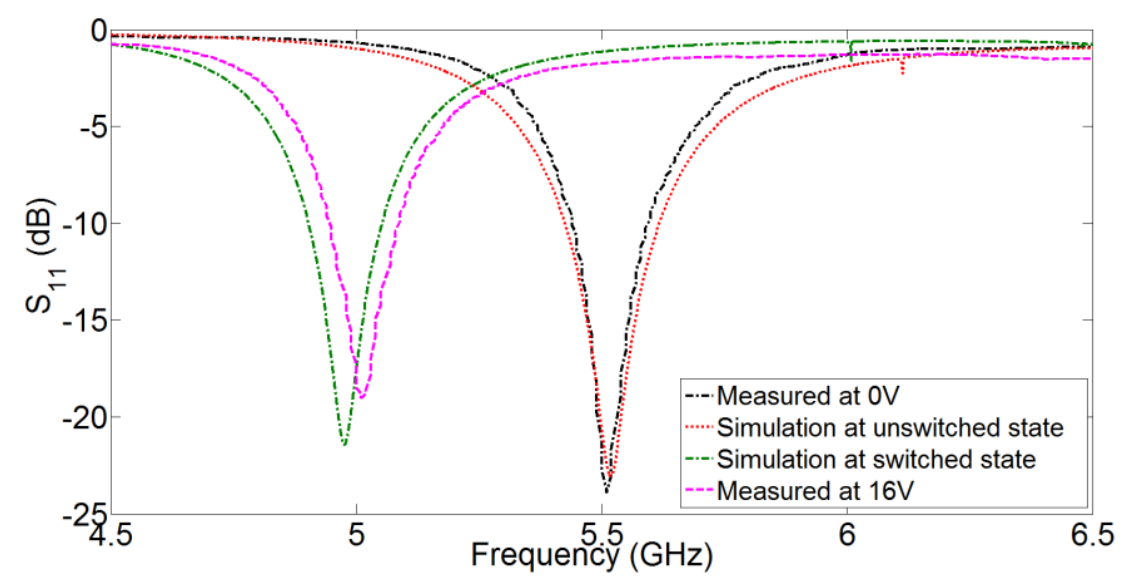

Figure 9. Comparison of measured and simulated $S_{11}$ at unswitched and switched state.

In order to provide a more accurate value for the effective permittivity in this state, the LC director simulation results shown in Figure 3 in the region between the electrodes and the ground plane are used to calculate an approximate value of the effective permittivity $\varepsilon_{e f f}$. CST simulations (Figure 3(a)-(b)) show that the highest microwave field is in this region and only low fields were found between the electrodes and beyond the edges. The effective permittivity was estimated by simply averaging the z-component of the dielectric constant in this high field region. This was found to be $\varepsilon_{e f f}=3.12$, which is slightly lower than $\varepsilon_{r \|}$ for E7. This value of $\varepsilon_{e f f}$ is then used in the CST simulation and the corrected resonant frequency is now 4.99GHz with the FBW of $8.8 \%$, and the insertion loss and return loss are $-23.6 \mathrm{~dB}$ and $-1.17 \mathrm{~dB}$, respectively. Compared with simulation using $\varepsilon_{r \|}=3.17$, the new resonant frequency is closer to the measured $S_{11}$ and shows more accurate agreement with the measured results.

\subsection{Comparison with the performance of other tunable filters based on LC}

Performance comparisons of the proposed filter with other tunable filters reported in the literature are summarised in Table 3. The comparison involves some characteristics of the filter, 
such as the centre frequency, FBW, tunability, insertion and return loss. As can be seen from Table 3, our work has better performance in narrower bandwidth, lower insertion loss, better return loss and the largest tuning.

Table 3. Comparison with previous tunable filters based on LC.

\begin{tabular}{|c|c|c|c|c|c|c|}
\hline $\begin{array}{l}\text { Tunable LC } \\
\text { filter }\end{array}$ & $\begin{array}{c}\text { Centre } \\
\text { Frequency } \\
(\mathrm{GHz})\end{array}$ & FBW & $\begin{array}{c}\text { Tunabilit } \\
\mathrm{y}\end{array}$ & $\begin{array}{l}\text { Insertion } \\
\text { Loss }(\mathrm{dB})\end{array}$ & $\begin{array}{c}\text { Return } \\
\text { Loss }(\mathrm{dB})\end{array}$ & LC \\
\hline [21] & 5 & $81.5-86.4 \%$ & $7.3 \% \%$ & $4.2-4.0$ & Unknown & $\begin{array}{l}\text { Unknown } \\
2.88-3.26\end{array}$ \\
\hline [22] & 5 & $22 \%$ & $6 \%$ & $6-9$ & $>7$ & $\begin{array}{c}\text { Unknown } \\
2.4-3.2\end{array}$ \\
\hline [27] & 5 & $12.2-12.6 \%$ & $7.3 \%$ & $3-6$ & $>18$ & $\begin{array}{c}\text { Merck } \\
\text { MDA-98- } \\
1602 \\
2.62-3.06\end{array}$ \\
\hline [28] & 5 & $41-50 \%$ & $6 \%$ & $>3$ & $>10$ & $\begin{array}{c}\text { K15 } \\
2.72-2.9\end{array}$ \\
\hline This work & 5 & $8.3-9.5 \%$ & $10 \%$ & $1.5-4.2$ & $>18$ & $\begin{array}{l}\text { Merck E7 } \\
2.72-3.17\end{array}$ \\
\hline
\end{tabular}

\section{Conclusion}

This paper has presented a general design method for microstrip tunable devices using LC technology at microwave frequency. The design method consists of a number of steps. The first is to model the desired structure as a lumped elements circuit, assuming the LC is an isotropic dielectric. The lumped element circuit modelling with ADS was then used to adjust the required resonant frequency and tuning range, and to perform a first optimisation of the 
dimensions for the device using ADS. The validity of the lumped element model can be verified comparing its results with those of CST where the LC is simulated as a homogeneous anisotropic material. This approximation can in turn be verified comparing the electric field from CST and the LC director distribution. The LC distribution follows the electric field distribution and is mostly vertical under and between the electrodes; thus, a uniform anisotropy is a valid approximation for the switched state. In the unswitched case, due to the uniform rubbing direction on the two substrates, the alignment of the LC is uniform and can be modelled accurately with CST. A second optimisation of the filter dimensions can now be made using CST to achieve the required centre frequency and tuning range, leading to the final design for the device.

The design method is demonstrated with the design and fabrication of a tunable microstrip bandpass filter, using an inverted microstrip structure consisting of IDCs and inductive loops to achieve a compact structure. The filter is designed to work at about $5 \mathrm{GHz}$ and the LC used is E7. The fabricated device is measured and shows a tuning range of $500 \mathrm{MHz}$ with a tunability of $10 \%$, in good agreement with the predictions from CST. Accurate finite element modelling of the LC switching is used to explain the small discrepancy in measured and simulated $S_{11}$ for the switched state. A more accurate value of the effective permittivity is estimated from the LC modelling and used in CST to give more accurate results that show a better agreement with the measured results. The comparison between this work and the literature shows that this filter has narrower bandwidth, larger tunability, lower insertion loss and a better return loss.

References: 
[1] Yaghmaee P, Karabey OH, Bates B, et al. Electrically tuned microwave devices using liquid crystal technology. Int. J. Antenn. Propag. 2013.

[2] Zhang XY, Chan CH, Xue Q, et al. RF tunable bandstop filters with constant bandwidth based on a doublet configuration. IEEE Trans. Ind. Electron. 2012; $59(2): 1257-1265$.

[3] Naglich EJ, Lee J, Peroulis D, et al. Switchless tunable bandstop-to-all-pass reconfigurable filter. IEEE Trans. Microw. Theory Technol. 2012; 60(5):1258-1265.

[4] Li JS, Liu H, Zhang L. Compact and tunable-multichannel terahertz wave filter. IEEE Trans. THz Sci. Technol. 2015; 5(4):551-555.

[5] Scarbrough D, Psychogiou D, Peroulis D, et al. Low-loss, broadly-tunable cavity filter operating at UHF frequencies. Microw. Symp. (IMS), IEEE MTT-S Int. 2015; Phoenix, AZ, USA.

[6] Nath J, Ghosh D, Maria JP, et al. An electronically tunable microstrip bandpass filter using thin-film Barium-Strontium-Titanate (BST) varactors. IEEE Trans. Microw. Theory Techn. 2005; 53(9): 2707-2712.

[7] Schuster C, Wiens A, Schmidt F, et al. Performance analysis of reconfigurable bandpass filters with continuously tunable center frequency and bandwidth. IEEE Trans. Microw. Theory Techn, 2017; 65(11): 4572-4583.

[8] Chaudhary G, Jeong Y, Lim J. Dual-band bandpass filter with independently tunable center frequencies and bandwidths. IEEE Trans. Microw. Theory Techn. 2013; 61(1):107-116.

[9] Fouladi S, Huang F, Yan WD, et al. High- Q narrowband tunable combline bandpass filters using MEMS capacitor banks and piezomotors. IEEE Trans. Microw. Theory Techn.2013; 61(1):393-402.

[10] Jiang H, Lacroix B, Choi K, et al. Ka and U band tunable bandpass filters using ferroelectric capacitors. IEEE Trans. Microw. Theory Techn. 2011:59(12): 30683075.

[11] Cheng C, Rebeiz GM. High-Q 4-6-GHz suspended stripline RF MEMS tunable filter with bandwidth control. IEEE Trans. Microw. Theory Techn.2011; 59(10):24692476.

[12] Chiou YC, Rebeiz GM. A tunable three-pole 1.5-2.2-GHz bandpass filter with bandwidth and transmission zero control. IEEE Trans. Microw. Theory Techn.2011; 59(11):2872-2878. 
[13] Anand A, Small J, Peroulis D, et al. Theory and design of octave tunable filters with lumped tuning elements. IEEE Trans. Microw. Theory Techn. 2013; 61(12):43534364.

[14] Li C, Bian Y, Li G, et al. A tunable high temperature superconducting bandpass filter realized using semiconductor varactors. IEEE Trans. Appl. Supercond. 2014; 24(5):15 .

[15] Wang X, Bao P, Jackson TJ, et al. Tunable microwave filters based on discrete ferroelectric and semiconductor varactors. IET Microw. Antennas Propag. 2011; 5(7):776-782.

[16] Liu X. Tunable RF and microwave filters. IEEE 16th Annu. Wireless and Microw. Techno. Conf. 2015; Cocoa Beach, FL, USA.

[17] Yazdanpanahi M, Mirshekar-Syahkal D. Investigation of coupling based on liquid crystal in two end-coupled microstrip resonators. In Telecommunications (IST), 2010 5th International Symposium on IEEE. 2010; 324-327.

[18] Yazdanpanahi M, Bulja S, Mirshekar-Syahkal D, et al. Measurement of dielectric constants of nematic liquid crystals at mm-wave frequencies using patch resonator. IEEE Trans. Instrum. Meas. 2010; 59(12):3079-3085.

[19] Goelden F, Gaebler A, Karabey O, et al. Tunable band-pass filter based on liquid crystal. IEEE German Microw. Conf. 2010; Berlin, Germany.

[20] Shen M, Huang Y, Shao Z. A tunable microstrip dual-mode bandpass filter based on liquid crystal technology. ICCPIEEE Int. Conf. 2015; Guilin, China.

[21] Cai L, Xu H, Pivnenko M. A tunable wideband microstrip bandstop filter based on liquid crystal materials. ICCP IEEE Int. Conf. 2014; Beijing, China.

[22] Jiang D, Chen H, Luo W. Tunable microwave filter using highly anisotropic liquid crystals. ICCP in IEEE Int. Conf. 2015; Guilin, China.

[23] Dai JW, Peng HL, Zhang YP, et al. A beam-steering array using liquid crystal phase shifter. Advanced Materials and Processes for RF and THz Applications (IMWSAMP) in IEEE MTT-S Int. Microw. Workshop Series, 2016; Chengdu, China.

[24] Zhao Y, Huang C, Qing AY, et al. A frequency and pattern reconfigurable antenna array based on liquid crystal technology. IEEE Photon. J. 2017; 9(3).

[25] Karabey OH, Gaebler A, Strunck S, et al. A 2-D electronically steered phased-array antenna with $2 * 2$ elements in LC display technology. IEEE Trans. Microw. Theory Techn. 2012; 60(5):1297-1306. 
[26] $\mathrm{Hu}$ W, Dickie R, Cahill R, et al. Liquid crystal tunable mm wave frequency selective surface. IEEE Microw. Wireless Compon. Lett. 2007; 17(9):667-669.

[27] Torrecilla J, Urruchi V, Sánchez-Pena JM, et al. Improving the pass-band return loss in liquid crystal dual-mode bandpass filters by microstrip patch reshaping. Materials. 2015; 7(6):4524-4535.

[28] Bernigaud JF, Martin N, Laurent P, et al. Liquid crystal tunable filter based on DBR topology. Proc. of the 36th Enropean Microw. Conf. 2006; Manchester, UK.

[29] Advanced Design System (ADS). Santa Clara: Keysight Technologies; 2012. https://www.keysight.com/en/pc-1297113/advanced-design-systemads?cc=GB\&lc=eng. [Access date: 02/06/2018]

[30] CST Mirowave Studio. Paris: CST Computer Simulation Technology AG; 2017. www.cst.com. [Access date: 02/06/2018]

[31] Yaghmaee P, Withayachumnankul W, Horestani AK, et al. Tunable electric-LC resonators using liquid crystal. Antennas and Propag. Soc. Int. Symp. 2013; Orlando, FL, USA.

[32] Hong JSG, Lancaster MJ. Microstrip filters for RF/microwave applications. New York (NY): John Wiley \& Sons; 2004.

[33] Bahl IJ. Lumped elements for RF and microwave circuits. Norwoood (MA): Artech house; 2003.

[34] Gevorgian S, Berg H. Line capacitance and impedance of coplanar-strip waveguides on substrates with multiple dielectric layers. 2001.

[35] Gupta K. C., Garg R., Bahl I., Bhartia P. Microstrip lines and Slotlines. Norwood (MA): Artech house; 1996.

[36] James, R, Fernandez, FA, Day, SE, Bulja, S, Mirshekar-Syahkal, D. Accurate modeling for wideband characterization of nematic liquid crystals for microwave applications. IEEE Trans. Microw. Theory Technol. 2009; 57(12): 3293-3297.

[37] Chandrasekhar S. Liquid crystals. Cambridge (UK): Cambridge University Press; 1992. 